\title{
Early damage and fatigue crack initiation at ambient and elevated temperatures in heat resistant austenitic steel
}

\author{
Jaroslav Polák ${ }^{1,2^{*}}$, Veronika Mazánová ${ }^{1}$, Roman Petráśs ${ }^{1,2}$ and Milan Heczko ${ }^{1}$ \\ ${ }^{1}$ Institute of Physics of Materials, Žižkova 22, 61662 Brno, Czech Republic \\ ${ }^{2}$ CEITEC IPM, Institute of Physics of Materials, Žižkova 22, 61662 Brno, Czech Republic
}

\begin{abstract}
Fatigue damage at ambient and at elevated temperature has been studied in advanced heat resistant austenitic steel. Cylindrical specimens were subjected to constant strain rate cycling and cycling with dwell in the tensile part of the cycle with different strain amplitudes. Surface relief evolution and initiation of surface and internal cracks were studied using high resolution SEM, FIB cutting, EBSD analysis, foil preparation and STEM imaging.
\end{abstract}

\section{Introduction}

Austenitic stainless steels are employed for construction of industrial equipment in a wide range of temperatures. Due to service loads and temperature variations they are subjected to fatigue damage. The nature of the fatigue damage depends substantially on the temperature. Recent studies at room temperature in 300 type steels [13] revealed that the cyclic strain localization is an important factor in the initiation and early growth of fatigue cracks. Cyclic plastic straining at room temperature is localized into persistent slip bands (PSBs) and repeated cyclic straining produces pronounced surface relief in the form of persistent slip markings (PSMs) at the locations where PSBs emerge on the surface [4]. PSMs consist of extrusions and intrusions. Preferentially intrusions represent crack-like defect from which fatigue cracks were proposed to initiate [4]. Alternative mechanism of crack initiation coming from the observations of the cracks in copper single and bicrystals $[5,6]$ is based on the nucleation of the cracks on the grain boundary or on the PSB/matrix interface by direct condensation of vacancies.

Study of crack initiation in materials subjected to elastoplastic cyclic loading at high temperatures concentrated preferentially to the superalloys and austenitic steels [7-9]. The effect of oxidizing atmosphere and the influence of stress and strain relaxations resulting in the creep damage has been recognized. Nevertheless the role of individual damage mechanisms which contribute to the decrease of the fatigue life in high temperature environment has not been distinguished.

In this contribution experimental study of the typical mechanisms related to the initiation of fatigue cracks in advanced heat resistant austenitic stainless steel at ambient and at high temperatures will be presented.

\section{Experimental}

Highly-alloyed austenitic stainless steel Sanicro 25 which has good mechanical properties at elevated temperatures was studied. The material was supplied by the Sandvik, Sweden in the form of a cylindrical rod of $150 \mathrm{~mm}$ in diameter. The chemical composition of the material in wt.\% was: $0.1 \mathrm{C}, 22.5 \mathrm{Cr}, 25.0 \mathrm{Ni}, 3.6 \mathrm{~W}, 1.5$ Co, 3.0 Cu, 0.5 Mn, 0.5 Nb, 0.23 N, 0.2 Si, balance Fe. Rough shape specimens were solution annealed at $1200{ }^{\circ} \mathrm{C}$ for one hour and cooled to room temperature in the air. The average grain size, found using the intercept method, was about $60 \mu \mathrm{m}$. Particles of Z-phase rich in $\mathrm{Nb}, \mathrm{Cr}$ and $\mathrm{N}$ are found preferentially at grain boundaries but also in the interior of the grains. Some larger Z-phase particles are arranged in chains approximately parallel to the axis of the rod.

Cylindrical specimens for room temperature cyclic straining had $8 \mathrm{~mm}$ in diameter and $12 \mathrm{~mm}$ in gage length. Specimens cycled at high temperature were button end with the diameter of $6 \mathrm{~mm}$ and the gage length $15 \mathrm{~mm}$. The axis of the specimen was parallel to the axis of the cylindrical rod. In the middle of the gage length of specimens cycled at room temperature a shallow notch of $0.4 \mathrm{~mm}$ in depth was ground. The area of the shallow notch was mechanically and electrolytically polished to facilitate the observation of crack initiation using optical microscopy (OM) and scanning electron microscopy (SEM). Also the whole gage length of cylindrical specimens subjected to high temperature cyclic loading was mechanically and electrolytically polished.

Specimens were subjected to symmetric tensioncompression strain cycle at ambient and at elevated temperature in an electrohydraulic computer controlled MTS test system. The strain was controlled and 
measured using axial extensometer having the $8 \mathrm{~mm}$ gage length. Split resistance furnace with three zones was used to achieve high temperatures. At room temperature the constant strain rate was $5 \times 10^{-3} \mathrm{~s}^{-1}$ and at temperature $700{ }^{\circ} \mathrm{C}$ the strain rate was $2 \times 10^{-3} \mathrm{~s}^{-1}$. Constant total strain amplitudes in the range of $(2.5-$ $7.0) \times 10^{-3}$ were applied. Stress and strain amplitudes were derived from the hysteresis loops during cycling. Plastic strain amplitude was determined as the half-width of the hysteresis loop at mean stress.

After cycling the specimen surface was inspected in detail using SEM-FEG Tescan Lyra3 XMU equipped with focused ion beam (FIB). FIB cuttings and SEM observations were used to reveal the shape of the extrusions, intrusions and starting cracks. In order to protect the shape of PSMs and cracks during FIB nanofabrication the surface of the specimen was in particular location first covered by a very thin sheet of platinum using electron deposition and later a thicker platinum layer was applied using ion deposition. FIB sectioning was performed perpendicular to the specimen surface and approximately perpendicular to the direction of PSMs. Final polishing of the cut was performed with low accelerating voltage of $5 \mathrm{kV}$.

Lamellae for TEM observations were prepared by fabricating craters from both sides of the future lamella. After extraction of the lamella from the specimen it was subsequently thinned repeatedly from both sides using low energy ions. Lamellae were then observed and characterized in STEM mode by JEOL JEM-2100F $\mathrm{S} / \mathrm{TEM}$ equipped with FEG electron source and working at $200 \mathrm{kV}$.

For the observation of the internal damage of specimens cycled at high temperature the longitudinal cut along the whole gage length was performed. The cut was mechanically polished to obtain the perfectly smooth surface. Longitudinal cuts were observed in SEM and corresponding EBSD images of the selected areas were performed as well.

\section{Results}

\subsection{Ambient temperature}

Room temperature cyclic straining results in formation of distinct PSMs in individual grains of the material. Fig. 1 shows the surface of a typical grain in a specimen subjected to the constant strain amplitude $4 \times 10^{-3}$ for 1850 cycles with the primary electron beam perpendicular to the surface. Low magnification picture shows large particles of Z-phase at grain boundaries and one larger grain with well-developed PSMs. One slip system is active producing several parallel PSMs. Some of them arise only in the centre of the grain and only a few of them reach one grain boundary. High magnification picture of the central part of the PSM shows wide PSM consisting of several parallel individual extrusions and intrusions. The exact 3dimensional information about the shape of the PSMs is missing. The image documents the localization of the cyclic strain only into several PSBs producing surface relief in the form of PSMs while the rest of the grain (matrix) is deformed only elastically thus no relief on the surface corresponding to the matrix is produced.

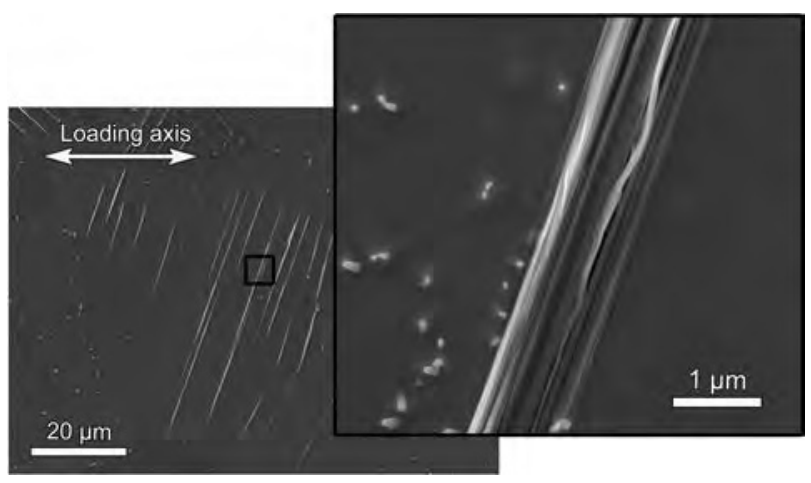

Fig. 1. Parallel PSMs in a grain after constant strain amplitude loading; $\varepsilon_{\mathrm{a}}=4 \times 10^{-3}$ for 1850 cycles.

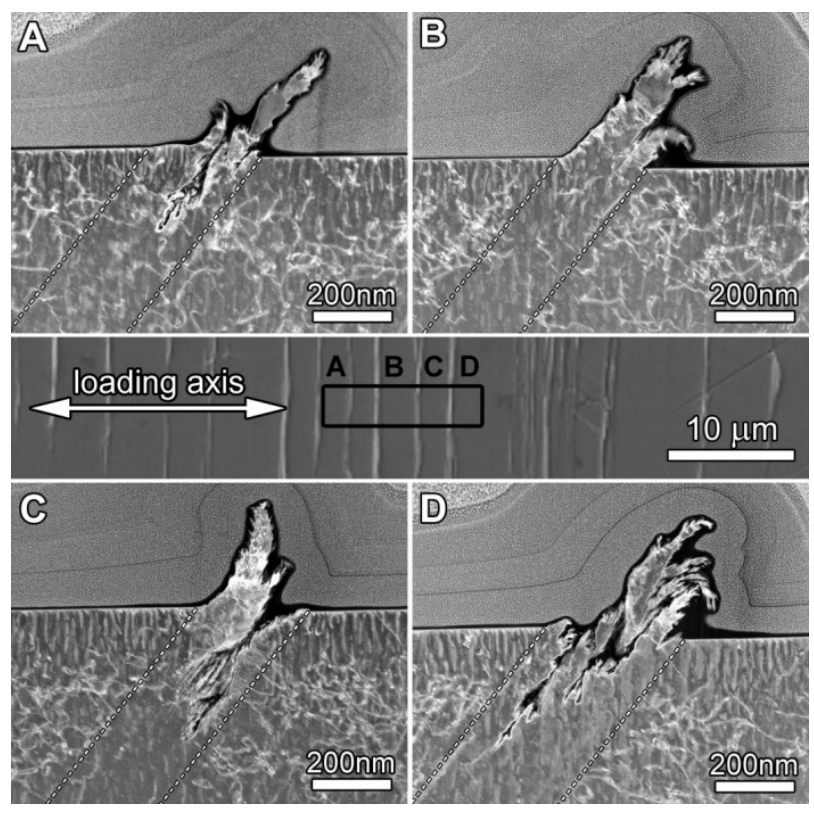

Fig. 2. Parallel PSMs in a grain of fatigued Sanicro25 steel and their profiles imaged using STEM of the foil prepared perpendicular to the surface. The surface was protected by electron (gray) and ion (not shown) deposition; dark field imaging.

3-dimensional information can be obtained using FIB sections perpendicular to the surface or fabricating thin lamella using FIB which can be later inspected in high resolution by TEM or STEM. Surface of a grain of the specimen cycled with constant strain amplitude $2.5 \times 10^{-3}$ to fracture is shown in the central section of Fig. 2. Several parallel PSMs, perpendicular to the stress axis are shown at low magnification. Black rectangle denotes the position of the lamella which was produced perpendicular to the surface. The low angle annular dark field STEM images of the lamella showing four parallel PSMs denoted A, B, C, and D are shown above and below. The profiles of all PSMs and the internal dislocation structure in the nearest vicinity of PSMs are distinctly displayed. The directions of the prospective persistent slip bands (PSBs) in the material are indicated by dashed white lines. PSMs consist of extrusions and 
intrusions or sharp valleys between two neighbour extrusions. The direction of extrusion and intrusion growth corresponds to the trace of the primary slip plane and the trace of the primary Burgers vector. Thin extrusions are often bent and sometimes peculiar shapes are produced. Intrusions represent very sharp notches and due to high plastic strain amplitude in the PSBs fatigue cracks start from the tip of the intrusions. Starting cracks can be recognized from the PSMs A, C and D. PSM B consists only of two extrusions and no crack has been initiated. Closer examination reveals that from the sharp valley between two extrusions a crack has a tendency to arise.

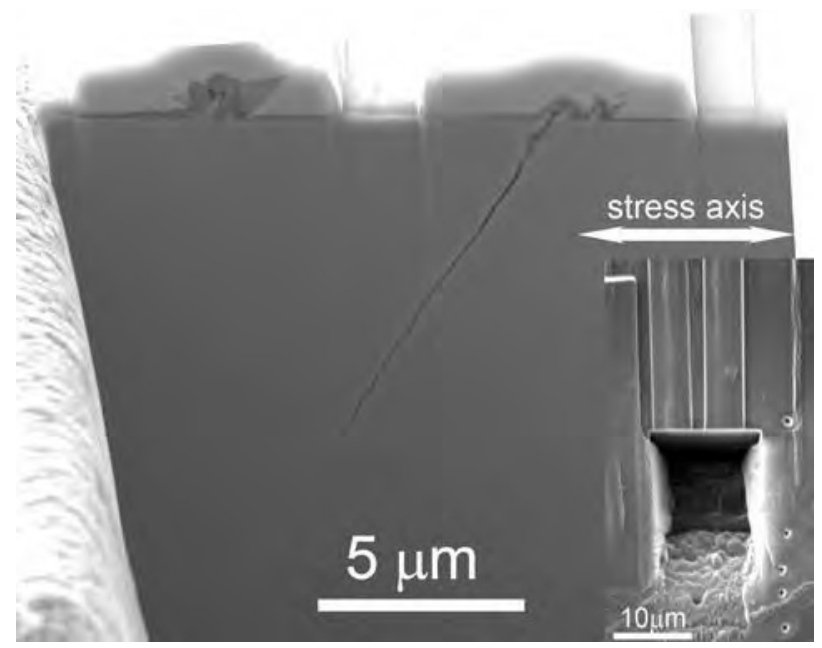

Fig. 3. FIB section of two PSMs covered by platinum straps with a crack starting from one of them; inset shows the location of the cut.

The paths of secondary short cracks starting from the PSMs were followed using FIB cutting. Fig. 3 shows the FIB cut with two PSM profiles on the surface of a specimen cycled with strain amplitude $3.5 \times 10^{-3}$ to fracture. The inset in the bottom right corner shows the position of the cut of two PSMs, each of them covered by the platinum layer. Both PSMs consist of extrusions and small intrusions. Only one intrusion/valley in the right PSM was deeper and from this intrusion/valley a fatigue crack developed and continued growing. The path of the fatigue crack initially followed primary slip plane but reaching approximately $10 \mu \mathrm{m}$ the direction of its growth deviated from primary slip plane with a tendency to become perpendicular to the stress axis.

\subsection{High temperature}

Cyclic loading at the temperature of $700{ }^{\circ} \mathrm{C}$ leads to premature initiation of fatigue cracks. Fig. 4 shows typical surface of the specimens of Sanicro 25 steel after high temperature cyclic straining. Fig. 4a shows the specimen surface at $10 \%$ of fatigue life of a specimen subjected to constant strain rate cycling and Fig. 4b shows the surface of fractured specimen subjected to constant strain rate cycling with $10 \mathrm{~min}$ dwell in maximum tension in a cycle.
The surface of the specimen shown in Fig. 4a is oxidized with oxide more developed at grain boundaries in the form of oxide extrusions. Preferentially grain boundaries on the surface perpendicular to the stress axis are oxidized more markedly and the oxide extrusions are cracked (see the detail of cracked oxide extrusion in the black inset).

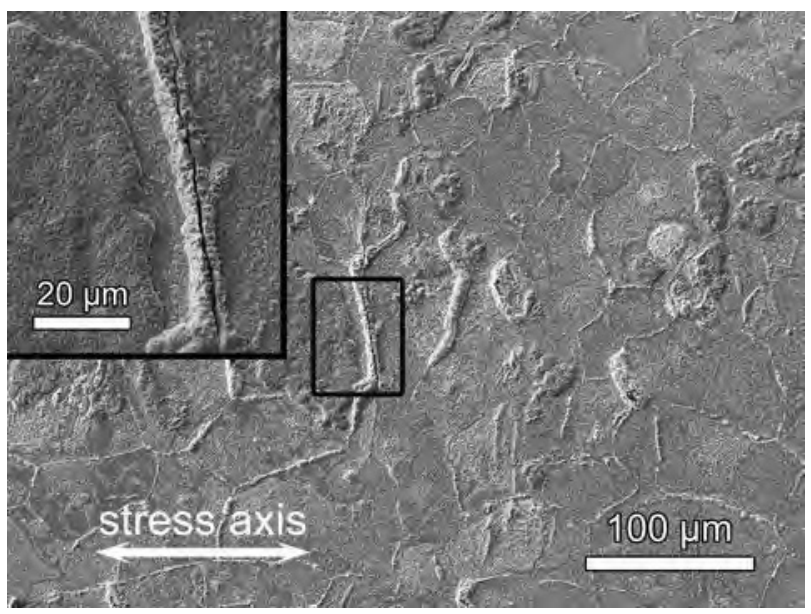

Fig. 4a. Surface of the specimen cyclically strained under constant strain amplitude loading at $700{ }^{\circ} \mathrm{C}$, (a) constant strain rate, $\varepsilon_{\mathrm{a}}=3 \times 10^{-3}, \mathrm{~N}=\mathrm{N}_{\mathrm{f}} / 10$.

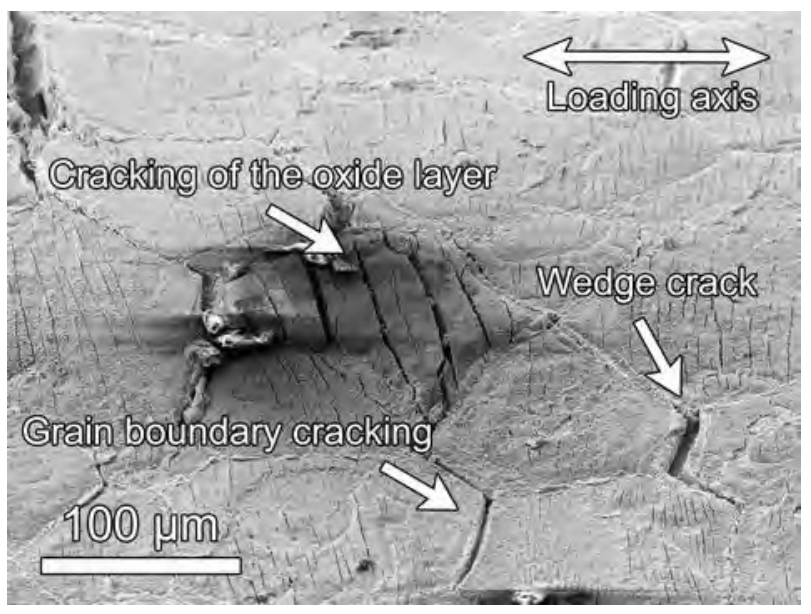

Fig. 4b. Surface of the specimen cyclically strained under constant strain amplitude loading at $700{ }^{\circ} \mathrm{C}, \varepsilon_{\mathrm{a}}=4 \times 10^{-3}, 10 \mathrm{~min}$ dwell in maximum tension, $\mathrm{N}=\mathrm{N}_{\mathrm{f}}$.

Specimen subjected to cycling with 10 min dwell up to fracture has thicker layer of oxide than specimens cycled with constant strain rate. Very high density of cracks developed in the oxide layer both inside the grains and in the grain boundaries. Numerous cracks in the grain boundaries perpendicular to the stress axis became wide open and in some of them the sliding during dwell period lead to the formation of wedge cracks Fig. 4b shows high density of cracks in the thick oxide layer on the whole surface and also cracked grain boundary approximately perpendicular to the loading axis. Another grain boundary was subjected to sliding and wedge crack has formed.

The profile of the grain boundary cracks in cycling with constant strain rate and in cycling with $10 \mathrm{~min}$ dwells is shown using FIB cuts perpendicular to the 
surface in Figs 5a and 5b, respectively. In constant strain rate cycling (Fig. 5a) the oxide layer far from the grain boundary is thin but close to the grain boundary the oxidation is very intensive. The oxide intrusion goes deep in the grain boundary and due to higher volume of the oxide also characteristic oxide extrusion protrudes along the grain boundary. The thick oxide layer is cracked. The crack is shallow in the case of Fig. 5a.

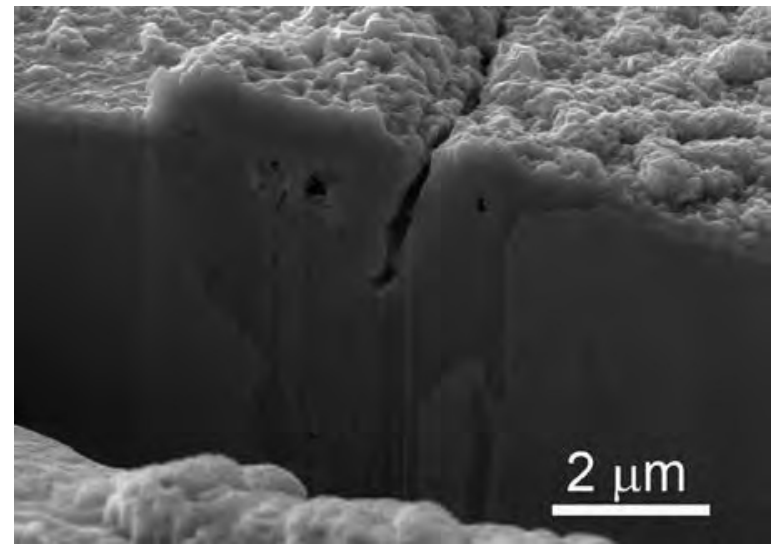

Fig. 5a. FIB section of the surface cracks along the grain boundaries in the specimen cyclically strained under constant strain amplitude loading at $700{ }^{\circ} \mathrm{C}$, constant strain rate, $\varepsilon_{\mathrm{a}}=3 \times 10^{-3}, \mathrm{~N}=\mathrm{N}_{\mathrm{f}} / 10$.

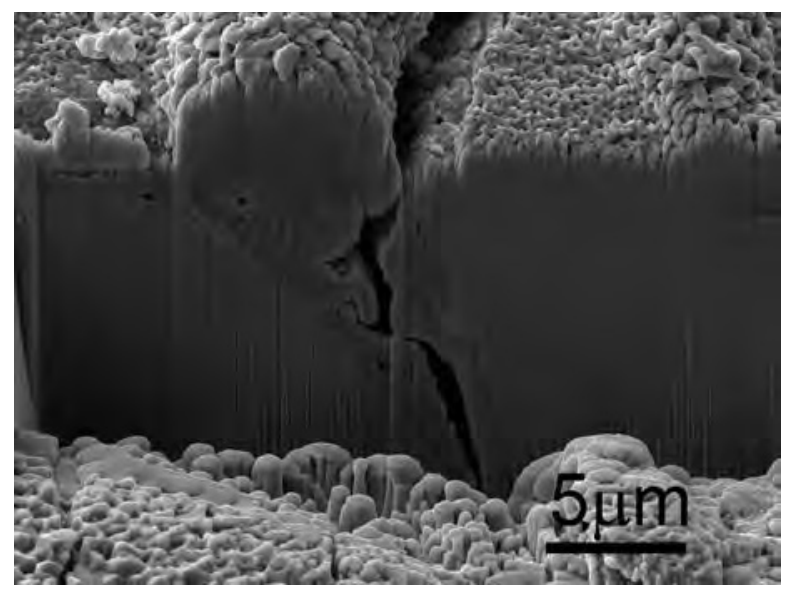

Fig. 5b. FIB section of the surface cracks along the grain boundaries in the specimen cyclically strained under constant strain amplitude loading at $700{ }^{\circ} \mathrm{C}$ with $10 \mathrm{~min}$ dwell in maximum tension, $\varepsilon_{\mathrm{a}}=4 \times 10^{-3}, \mathrm{~N}=\mathrm{N}_{\mathrm{f}}$ (secondary crack).

In cycling with dwells the thickness of the oxide layer on the surface of grains and even more the height of the oxide extrusion are substantially larger (Fig. 5b). The oxide intrusion along the grain boundary extends deep in the material. The oxide is cracked up to the tip of the oxide intrusion. Fig. 5 illustrates that the oxidation of the grain boundaries in cooperation with the cyclic loading which stimulates the cracking of the oxide intrusion contribute to the early initiation and growth of fatigue cracks.

In cyclic straining with dwells fatigue cracks initiate not only on the surface of the specimen but also in the interior. Fig. 6 shows the longitudinal cut of the specimen fatigued up to fracture with constant strain amplitude and $10 \mathrm{~min}$ dwell in the tensile maximum of the cycle. In addition to the principal macroscopic crack starting from the surface and a number of small surface cracks also several small cracks having the length of around $200 \mu \mathrm{m}$ in the interior of the specimen has arisen. The interior cracks could be found in all locations on the surface of the longitudinal cut but higher density of the interior cracks was found close to the tip of the macroscopic crack. The origin of these cracks was studied by searching initial stages of their formation. Fig. 7 shows junction of three grains with number of small voids along the grain boundaries. This observation allows us to consider the mechanism of internal crack formation during elastoplastic cyclic loading with dwells. Cyclic loading produces high number of point defects, predominantly vacancies. In case of cyclic loading with dwells the dislocation density can substantially decrease during high temperature dwell. Vacancies have high probability to coalesce. Coalescence of vacancies along the grain boundaries and further condensation of the voids leads to the internal grain boundary cracks. The internal grain boundary cracks can be linked with the principal crack and contribute to the increase of the crack growth rate in cycling with dwells.

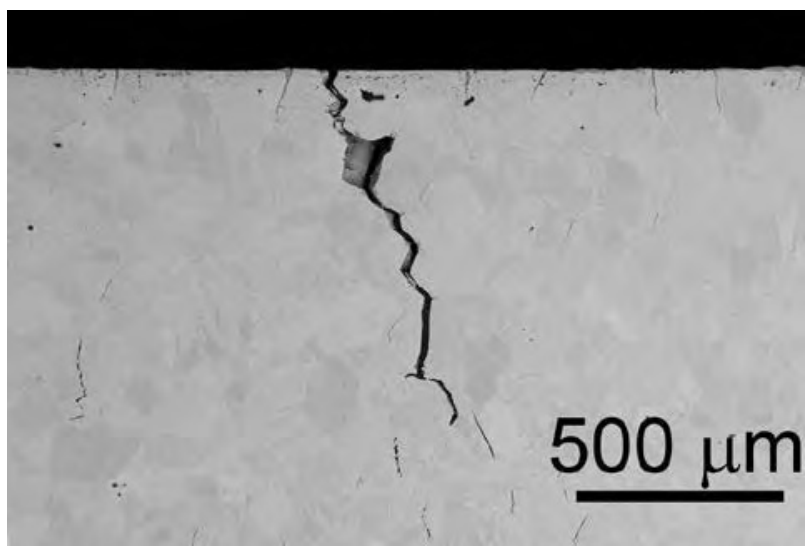

Fig. 6. Longitudinal cut of the specimen cyclically strained under constant strain amplitude at $700{ }^{\circ} \mathrm{C}$ with constant strain rate and $10 \mathrm{~min}$ dwell in maximum tension, $\varepsilon_{\mathrm{a}}=4 \times 10^{-3}, \mathrm{~N}=\mathrm{N}_{\mathrm{f}}$.

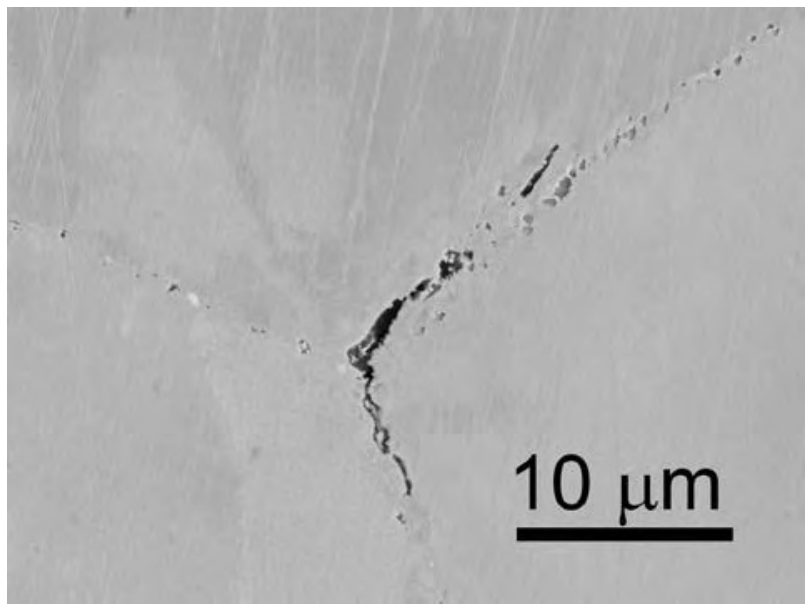

Fig. 7. Longitudinal cut of the specimen cyclically strained under constant strain amplitude loading at $700{ }^{\circ} \mathrm{C}$ with constant strain rate and $10 \mathrm{~min}$ dwell in maximum tension, $\varepsilon_{\mathrm{a}}=4 \times 10^{-3}$, $\mathrm{N}=\mathrm{N}_{\mathrm{f}}$. 
Internal crack on the longitudinal cut of the specimen cycled with dwells in the tensile part of the cycle is shown in Fig. 8a. Corresponding EBSD image of the identical area displayed in Fig. 8a is shown in Fig. 8b. It is evident that internal crack follows grain boundaries. Moreover, it is not oxidized and therefore the hypothesis assuming that it originates due to production and coalescence of internal cavities on the grain boundaries is very probable.

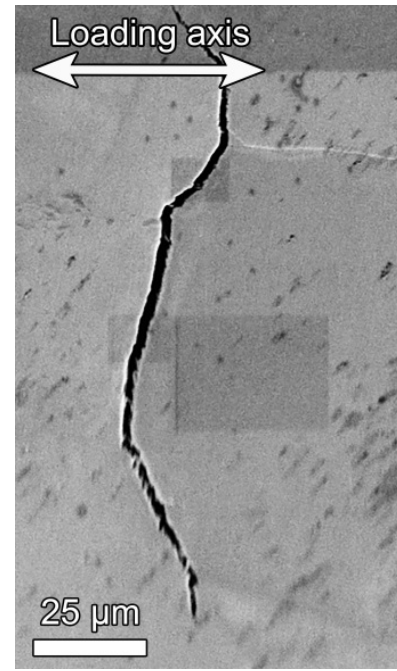

(a)

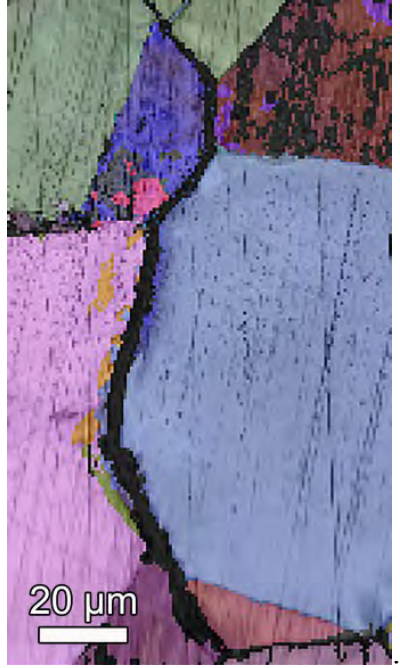

(b)
Fig. 8. Longitudinal section of the specimen subjected to constant strain rate and $10 \mathrm{~min}$ dwell in maximum tension showing internal crack, (a) SEM image, (b) EBSD image.

\section{Discussion}

Present results show clearly how the temperature influences fatigue crack initiation in high resistant austenitic steel. Two extreme cases were investigated i.e. room temperature and the highest projected temperature for this sort of austenitic stainless steel $\left(700^{\circ} \mathrm{C}\right)$. At room temperature in agreement with previous results [10-12] cyclic strain is highly localized. While the major volume of the grains undergoes only elastic straining the plastic strain amplitude in the bands of intensive cyclic slip (in PSBs) is high. As a result pronounced surface relief arises at locations where PSBs emerge on the surface of the grains and PSMs are produced. PSMs consist of extrusions and intrusions but observation of the surface using even high resolution SEM (see Fig. 1) or even AFM [1] cannot yield a true profile of the PSMs. This is due to very fine structure of the PSMs (see Fig. 2) and also due to the inclination of the primary slip plane to the surface. This inclination is in most cases close to 45 degrees. Therefore even very fine tips of the AFM cannot reproduce the true shape of the PSMs [1]. Either FIB cutting and SEM imaging or the production of the site-specific foil for TEM or STEM using FIB nanofabrication can yield the details of the extrusions and intrusions. In the center of Fig. 2 the rectangle indicates the location where the foil for transmission electron microscopy was produced using FIB machining. The foil was thinned and observed both in SEM and in STEM. The images in STEM give much more details and thus the profiles of all four PSMs are shown in Fig. 2. The images reveal complicated shapes of the PSMs in austenitic steel contrary to e.g. simple hill shapes in the grains of copper polycrystal [12]. PSM profiles are formed preferably by extrusions but parallel to extrusions (PSM C and D) or in between two extrusions (PSM A) crack-like intrusion appears. From sharp intrusions stage I cracks start and grow parallel to the primary slip plane. Fig. 3 shows the crack path of the stage I crack starting from one of two PSMs on the surface of the grain. Growing crack absorbed majority of applied plastic strain and as a result the other PSM did not produce an appreciable crack. The growing crack close to the surface followed primary slip plane and becoming longer its path has changed to approach a direction perpendicular to the stress axis.

Formation of the PSM, i.e. the growth of extrusions and formation of intrusions, have been recently treated quantitatively $[4,13]$. The driving force for extrusion and intrusion formation is the redistribution of matter between the PSB and the neighbor matrix. Continuous production of point defects during cyclic plastic straining in PSB and their systematic migration from the PSB to the matrix results in surplus of matter in PSB and deficiency of matter in a thin sheet close to the $\mathrm{PSB} /$ matrix interface. It provokes internal compression in PSB and internal tension in a thin sheet at the $\mathrm{PSB} /$ matrix interface. The relaxation of internal compressive stresses due to cyclic plastic straining leads to formation of extrusions in locations where PSBs emerge on the surface. At a later stage [13] relaxation of internal tensile stresses results in a parallel intrusion at the $\mathrm{PSB} /$ matrix interface. Originally this mechanism was derived for ladder-like dislocation structure of a PSB in copper. However, it can work in any PSB in which plastic strain amplitude is an order or two orders higher than plastic strain amplitude in the neighbor matrix.

Fatigue damage at the temperature of $700{ }^{\circ} \mathrm{C}$ is substantially affected by temperature. In cyclic loading at elevated temperature the oxidation of the surface is not homogeneous. In the interior of the grains a thin protective layer of the chromium oxide is formed that prevents further oxidation of the material. Preferential formation of the chromium oxides and nucleation of $\mathrm{M}_{23} \mathrm{C}_{6}$ chromium carbides at the grain boundaries [14] decreases the chromium content along the grain boundaries. As a result the grain boundaries are preferentially oxidized. An oxide extrusion and oxide intrusion arise at the grain boundaries. As soon as the oxide layer becomes thicker it is easily cracked in tensile part of the cycle. Preferentially the grain boundaries whose directions on the surface are approximately perpendicular to the loading axis become cracked. The cracking of the oxide extrusion and intrusion allows the access of oxygen to the crack tip. The alternating oxidation of the crack tip and cracking of the oxide layer is an effective mechanism of the early growth of initiated fatigue cracks in our austenitic steel. This mechanism is similar to that observed in high temperature cyclic straining of superalloys [15].

Introduction of dwells in tensile maximum of a cycle has pronounced effect on the formation of surface cracks 
but results also in the formation of internal cracks [16]. Initiation of internal cracks has been observed only in high temperature cycling with dwells [16]. Cyclic plastic straining produces point defects $[4,13]$. Due to high formation energy only a small fraction of produced point defects are interstitials. Since their migration energy is very low they disappear rapidly at edge dislocations. Vacancy type defects are produced in much higher concentration. They arise by annihilation of two opposite edge segments on neighbor slip planes or by nonconservative motion of the jogs on screw dislocations in the form of unite dipoles. The unite dipoles can disintegrate and vacancies, divacancies and trivacancies can migrate and interact. Fraction of vacancies is annihilated at edge dislocations. There is however high probability of an encounter of two vacancy type defects and formation of immobile nucleus of a void. This void can grow by absorbing other vacancies. Grain boundaries which are rich in Z-phase and $\mathrm{M}_{23} \mathrm{C}_{6}$ carbides are preferred sites for the formation of a number of small voids. Once the voids are present along the grain boundary they can be linked together and internal cracks are produced.

It was proven experimentally that the internal cracks start to be formed on the grain boundaries by formation of voids along the grain boundary (Fig. 7). The crack path of internal cracks following grain boundaries was corroborated by EBSD images of the area containing an internal crack (Fig. 8). In this regard it is interesting to note that some authors $[5,6]$ propose a similar mechanism for initiation of fatigue cracks in room temperature cyclic straining without dwells. However the conditions in room temperature cyclic straining are less favorable for the accumulation of point defects since dislocation density is high and point defects created during cyclic plastic straining migrate preferentially to dislocations.

\section{Conclusions}

Constant amplitude cyclic straining of advanced austenitic stainless steel at room temperature and temperature $700{ }^{\circ} \mathrm{C}$ and subsequent analysis of the surface and internal fatigue damage led to the following conclusions

(i) High degree of cyclic lip localization is present in room temperature cyclic plastic straining.

(ii) Cyclic plastic strain is localized in PSBs and pronounced PSMs consisting of extrusions and intrusions are produced in locations where PSBs emerge on the surface.

(iii) Intrusions represent crack-like defects and true fatigue crack is initiated at its tip and continues to grow in stage I parallel to the primary slip plane.

(iv) High temperature cyclic straining results in preferential oxidation of the grain boundaries producing oxide extrusions and intrusions.

(v) Oxide extrusions and intrusions on the grain boundaries perpendicular to the stress axis on the surface are cracked and oxygen is admitted to the tip of the oxide intrusion. Repeated oxidation and cracking of the oxide leads to the early growth of the crack along the grain boundary.

(vi) Introduction of dwells in tensile maximum of the cycle results in grain boundary sliding and formation of wedge cracks and simultaneously to the formation of internal cracks in the whole volume of the cyclically deformed material. Internal cracks can link with the growing macroscopic crack and accelerate its growth.

The equipment used in the present project was provided by the Large Infrastructures for Research, Experimental Development and Innovation - project IPMinfra, LM2015069. The support by the Ministry of Education, Youth and Sports of the Czech Republic under the project CEITEC 2020 (LQ1601) is acknowledged.

\section{References}

1. J. Man, M. Valtr, M. Petrenec, J. Dluhoš, I. Kuběna, K. Obrtlík, J. Polák, Int. J. Fatigue 76, 11 (2015)

2. L. Signor, P. Villechaise, T. Ghidossi, E. Lacoste, M. Gueguen, S. Courtin, Mater. Sci. Eng. A 649, 239 (2016)

3. J.W. Pegues, M.D. Roach, N. Shamsaei, Mater. Sci. Eng. A 707,657 (2017)

4. J. Polák, J. Man, Int. J. Fatigue 91, 294 (2016)

5. L.L. Li, P. Zhang, Z.J. Zhang, H.F. Zhou, S.X. Qu, J.B. Yang, Z.F. Zhang, Acta Mater. 73, 167 (2014)

6. H.W. Hoppel, P. Goik, C. Krechel, M. Goken, J Mater.Res. 32(23), 4276, (2017)

7. S.D. Antolovich, E. Rosa, A. Pineau, Mater. Sci. Eng. 47, 47 (1981)

8. J. Weiss and A. Pineau, Metall. Trans. A, 24, 2247, (1993)

9. A. Pineau, S.D. Antolovich, Materials at High Temperatures 32(3), 298 (2015)

10. J. Polák, R. Petráš, G.C. Chai, V. Škorík, Mater. Sci. Eng. A 658, 221 (2016)

11. J. Polák, V. Mazánová, I. Kuběna, M. Heczko, J. Man, Metall Mater Trans A 47, 1907 (2016)

12. J. Polák, V. Mazánová, M. Heczko, I. Kuběna, J. Man, Fatigue Fract. Eng. Mat. Str. 40, 1101, (2017)

13. J. Polák, J. Man, Mater. Sci. Eng. A 596, 15 (2014)

14. V. Mazánová, M. Heczko, J. Polák, Int. J. Fatigue (2018) (in print)

15. L. Viskari, H.M. Hörnqvist, K.L. Moore, Y. Cao, K. Stiller, Acta Mater 61, 3630 (2013)

16. R. Petráš, J. Polák, Mater. Sci. Eng. A (2018) (in print) 